\title{
ELEMENTOS DE LA IDENTIDAD EN EL ESPACIO PÚBLICO La experiencia de Patio Bonito, un barrio sin proyecto
}

\author{
Juliana Arboleda Kogson \\ Universidad Politécnica de Catalunya \\ Director de tesis: Xavier Monteys \\ kogson@hotmail.com
}

\section{RESUMEN}

El espacio público que se ha desarrollado en la arquitectura de los barrios populares posee diferentes características que lo hacen singular entre los muchos otros que pueden verse en una metrópoli como Bogotá. La dimensión de la calle, la cantidad y calidad de los vacíos urbanos o la modificación de las alturas de las viviendas son algunas de las premisas que suele aparecer en el análisis de lo construido por los pobladores en este tipo de barrios. Sin embargo, en Patio Bonito, a estas premisas iniciales es necesario sumarle otro tipo de elementos que complementan y transforman lo público (la decoración de las fachadas, la extensión de las aceras hacia el interior de las plantas bajas, la aparición de vehículos de comercio y transporte ambulante) que tienen una incidencia directa en lo cotidiano. De esta manera se busca analizar todos los elementos que le otorgan identidad al barrio y que lo distinguen de muchos otros existentes con características similares.

Palabras clave: Espacio público, barrios populares, mejoramiento urbano, Bogotá

\section{ABSTRACT}

The public space developed in popular architecture has different characteristics that make it singular among the many others that can be seen in a metropolis like Bogota. The dimension of the street, the quantity and quality of the urban lacks or the modification of the heights of the houses are some of the premises with which it usually appears in the analysis of what the inhabitants built in this type of neighborhoods. However, in Patio Bonito, to these initial premises it is necessary to add another type of elements that complement and transform the public (the facades decoration, the extension of the low floors, the appearance of artifacts of commerce and ambulant transport) that has a direct incidence in the formation of the everyday. In this way, it is sought to analyze all the elements that grant identity to the neighborhood and that characterize it of many others existing with similar characteristics.

Key words: Public space, popular neighbor, urban improvement, Bogotá 
"El plano de la ciudad es más la obra del tiempo que el resultado de la acción de un arquitecto" es una de las frases más conocidas del arquitecto francés Leonce Reynaud y con la que se quiere abrir, en este artículo, la discusión acerca de los procesos de construcción y transformación del espacio público en una metrópoli como Bogotá y concretamente, las estrategias para la construcción de lo público en los barrios populares. Bogotá es una ciudad que se extiende $33 \mathrm{~km}$ de norte a sur y $16 \mathrm{~km}$ de este a oeste, según las cifras del CENSO de 2005, y que se ha construido en diferentes períodos de tiempo, con diferentes tramas y formas urbanas y en la que ha participado tanto la administración pública, como los promotores privados y los mismos habitantes. Actualmente es fácil identificar, a grandes rasgos, en un plano o en una vista aérea la trama fundacional de manzanas cuadradas del centro de la ciudad; los barrios proyectados durante la segunda mitad del siglo veinte en los que tanto las viviendas como el espacio público estaban pensados y ejecutados de manera sincrónica; los conjuntos cerrados de bloques de vivienda que han proliferado desde los años noventa (Parias D, 2012: 83) en los que las viviendas se aíslan completamente de la calle para enfatizar la seguridad ciudadana; y los barrios populares situados en las zonas que por sus condiciones topográficas están clasificadas como no urbanizables.

De las diferentes zonas, tramas y condiciones urbanas existentes puede decirse que, hasta el momento de la aparición del conjunto cerrado como forma urbana, la ciudad producía un tipo de espacio público en el que tanto el "lleno" como el "vacío" eran complementarios. Esto no quiere decir que los espacios construidos no tuvieran limitaciones o problemas, pero en cambio estaban abiertos al mejoramiento constante de su forma y de los elementos que los componían. La llegada de los conjuntos cerrados marca un antes y un después en la concepción y formación de nuevos desarrollos urbanos donde la distribución interior de la vivienda ha intentado mejorarse, pero en cambio el espacio público ha perdido condiciones, puesto que se ha desligado de lo doméstico. En este mismo sentido Clemencia Escallón plantea que "parece como si recientemente la dinámica de intervención en el ámbito de lo popular, en la solución al déficit, tanto cuantitativo como cualitativo, hubiera tenido una gestión inadecuada. La especialización de los actores y su desarticulación en el proceso de producción de la vivienda, sobre todo en la de bajo costo, dificulta la generación de productos de calidad. Cada uno responde por una parte del proceso y difícilmente se logra una coordinación que genere sinergias adecuadas." (Escallón, 2008:257).

El tipo de urbanismo que se identifica en los barrios populares es diferente al que puede observarse en el resto de la ciudad puesto que comparte los problemas de falta de diseño "a medida" de lo público o la negación de la calle en el conjunto cerrado antes relatada, sumado a la falta de recursos económicos para su desarrollo. Sin embargo, a pesar que el panorama es poco alentador, existen casos donde la escasez de recursos ha llevado a los actores a optimizar lo existente y realizar un mejoramiento de las infraestructuras con el paso del tiempo. Este es el caso de Patio Bonito un barrio en el que, como veremos a continuación, se ha realizado un crecimiento progresivo de las viviendas pero también un cambio en la calidad y las condiciones de lo público que va desde las acciones propias del trabajo en las infraestructuras, como la pavimentación de las calles o la conformación de alamedas, hasta la ubicación del comercio en arterias principales en donde han aparecido artefactos de comercio y/o transporte ambulante que modifican el uso de la calle. Un tipo de acciones que han motivado el encuentro y la convivencia, y que ha mejorado las condiciones urbanas, que ha sido posible gracias a la coordinación de los residentes a la hora de realizar acciones sobre los espacios colectivos y que han sido recogidos en los documentos editados por la Fundación Corpogés como parte del balance de los procesos de planeación del territorio. (Fundación Social, 2010 y García, 2011)

Se trata de encontrar los elementos que le dan identidad al barrio y que lejos de ser simples anécdotas, dan una pista acerca de cómo debería ser el tratamiento de lo público en otros lugares de la ciudad principalmente en las zonas de nuevos desarrollos. Hasta ahora la arquitectura de los barrios populares ha sido vista como un tipo especial de arquitectura que debe ser mejorada, la mayor parte de las veces por juicios de valor estético, pero en pocos casos se han resaltado sus valores para extraer lecciones. Es importante tener presente que gran parte de las acciones realizadas por los pobladores en los barrios populares son más eficientes a la hora de usar el espacio público puesto que parten de la necesidad pero también del ingenio, contrapuesto a lo que se proyecta sobre el papel, sin partir de una vivencia concreta, en las mesas de trabajo de la administración pública. Estos últimos son los responsables de las nuevas zonas de crecimiento de la ciudad en donde la vivienda ha mejorado la distribución interior, a pesar de la reducción progresiva de los metros cuadrados de uso, pero en donde faltan ideas para realizar un espacio público que sobrepase las necesidades propias de las infraestructuras para convertirse en espacios de intercambio social. Se busca pues dar pistas de cuáles son los elementos que dan identidad a los barrios populares y que pueden extrapolarse fuera de ellos. 

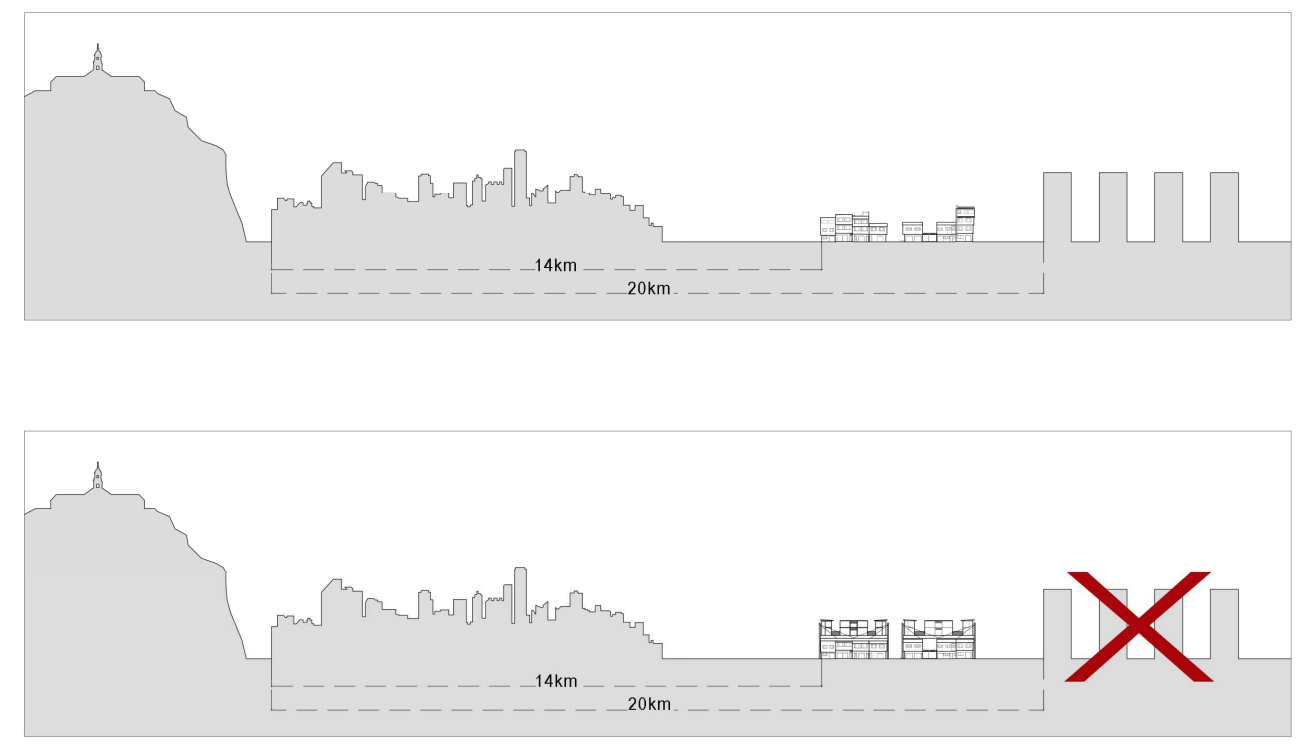

Ubicación de Patio Bonito con respecto al centro de la ciudad y con respecto a la nueva zona de urbanización "Ciudad Verde" Fuente: Juliana Arboleda Kogson, 2016

\section{PATIO BONITO: UN BARRIO SIN PROYECTO}

"Patio Bonito. Un barrio sin proyecto" es el título de la tesis defendida en enero de 2016 (Arboleda, 2016) en la que se realiza un análisis de los procesos de construcción y transformación en términos urbanos y arquitectónicos de un barrio singular, que por razones diversas se destaca entre muchos otros que se han desarrollado en las zonas populares de Bogotá bajo unas circunstancias prácticamente iguales. Patio Bonito es singular puesto que ha sabido superar la problemática de la urbanización inicial para pasar a la construcción de un tipo de calle y de un espacio público que se realiza de manera conjunta con las viviendas y por los mismos actores, sus pobladores, quienes se han empeñado en mejorar las infraestructuras con el paso del tiempo.

Pero para entender mejor cuales son las características del barrio y comprender las herramientas que se han desarrollado para su progreso es necesario primero conocerlo. Patio Bonito se asienta en el suroccidente de la capital, en la localidad de Kennedy, sobre un terreno no urbanizable al costado del rio Bogotá. Su ubicación no es espontánea puesto que está completamente ligada a la aparición en 1972 de la central de abastos "CORABASTOS" la cual, desde la fecha hasta la actualidad, es la mayor infraestructura para la compra y venta de víveres de la ciudad. La central de mercado por su envergadura ha sido el motor de desarrollo de la zona propiciando la aparición de una serie de urbanizaciones para dar acogida a los trabajadores. La colección de viviendas y barrios que se forman en la zona es diversa y comprende ejemplos tan variados como "Ciudad Kennedy", el proyecto "Kennedy Experimental" o el conjunto "Timiza", urbanizaciones promovidas por la Administración Pública, hasta barrios de origen informal como es el caso de Patio Bonito. Es importante aclarar en este punto que, a pesar que las obras en Patio Bonito se inician sin licencia de obra y sobre terrenos que no cumplían con óptimas condiciones topográficas, se urbaniza bajo los lineamientos del plano de loteo elaborado por la empresa Inversiones Samudio S.A en el que es posible observar la distribución de los lotes y de las vías principales. Sin embargo el hecho de realizarse por fuera de las zonas urbanizables de la ciudad lo deja en un vacío legal, en cuanto a la normativa específica de planeamiento urbano, por lo que el barrio se edifica según las ideas e intuiciones de cada habitante y que es visible en la diversidad de tipologías de vivienda, de crecimientos en altura y de secciones de la calle. 

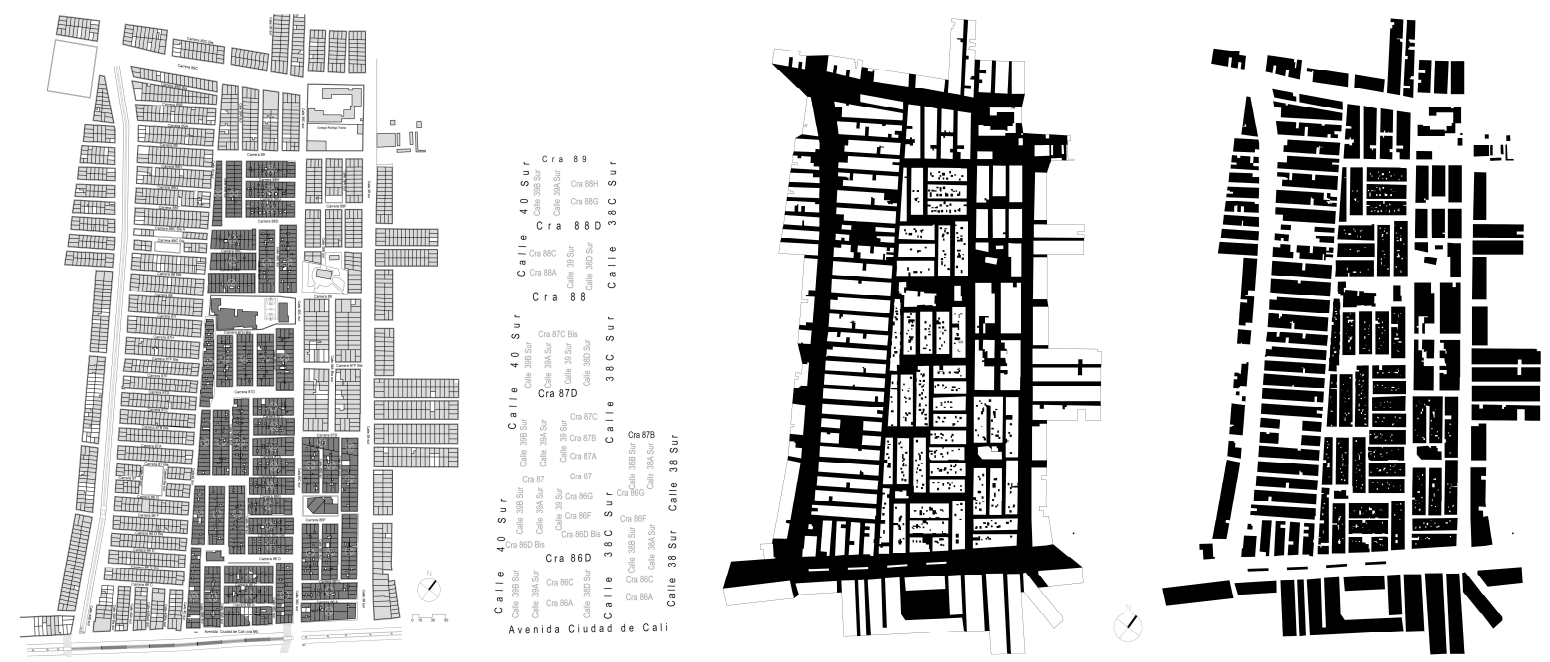

Cuatro planos de Patio Bonito: Plano del parcelario, plano de las calles y planos de llenos y vacíos urbanos Fuente: Juliana Arboleda Kogson, 2016

El barrio se extiende sobre 12.46 hectáreas y se caracteriza por la continuidad espacial de la edificación y la inexistencia de espacios libres diferentes a las calles y por la ausencia de espacios verdes tanto de parques como de pequeños antejardines. El espacio público se reduce entonces a la calle y a los vacíos urbanos que aparecen cuando una de ellas, por una condicionante topográfica o por la aparición de los límites de la urbanización, se ensanchan ligeramente. Sin embargo, podemos decir que la continuidad de la calle lo dota de personalidad si se analiza la homogeneidad que se produce en el alzado. La trama urbana distribuye las parcelas en manzanas alargadas, de diferente orientación y forma, que parecen provenir más de una antigua plantación de flores, tan presentes en el paisaje de la sabana de Bogotá, que de un planeamiento urbano. De esta manera se obtienen cuarenta y tres manzanas longitudinales que agrupan 1050 parcelas, de las cuales sólo nueve se encuentran actualmente sin edificar. Si bien todas las manzanas son longitudinales, la orientación varía de unas otras, siempre a noventa grados, por lo que no es posible definir una malla ortogonal. La calle que se construyen entre una manzana y otra diferencia su amplitud según el tipo de tráfico que circule, es decir, son más anchas las que se ubican en los ejes comerciales por las que transitan coches, autobuses y personas, que las que son solamente peatonales. Cada una de ellas define una unidad espacial diferente debido a que la anchura condiciona el tipo de comercio a situarse en planta baja, la altura de la edificación y, por tanto, el desarrollo interior de la vivienda. Las manzanas alargadas son de una o dos crujías, dependiendo de su posición con respecto a los límites, con parcelas rectangulares de $6,00 \mathrm{~m} \times 12,00 \mathrm{~m}\left(72 \mathrm{~m}^{2}\right)$ o de $7,00 \mathrm{~m} \times 14,00 \mathrm{~m}\left(98 \mathrm{~m}^{2}\right)$ que ocupan las manzanas modificando su cantidad. La altura de las edificaciones es variable, encontrando viviendas de planta baja, planta baja más una altura, planta baja más dos alturas y así hasta llegar a seis plantas con respecto al nivel de la calle.

De manera sorprendente la pluralidad de soluciones que encontramos en el barrio ha conformado un paisaje urbano sugestivo que ha sido advertido por algunos académicos como René Carrasco Rey o Clemencia Escallón pero que hasta ahora no había sido muy documentado. La primera publicación en la que se advierte las características de la calle y de los perfiles del barrio puede encontrarse en la recopilación realizada por Andrés Borthagaray en el libro "GGanar la calle! Compartir sin dividir" (Borthagaray, 2009) un documento en el que se analizan diferentes tipos de calles en doce ciudades América Latina que van desde lo que sucede en la Av. Insurgentes (México) o en el Paseo Reforma, las diferentes experiencias en Curitiva o la Av. Paulista (Sao Paulo) entre muchas otras. En el libro, que hoy puede consultarse en la web del proyecto, se recoge una sección de Patio Bonito en la que se hace evidente la disparidad en las alturas de las viviendas y los anchos de las diferentes vías y en la que se observa cómo se construye y se transforma la sección de la calle con las sucesivas modificaciones realizadas en la vivienda que alteran el cajón que enmarca la perspectiva. 

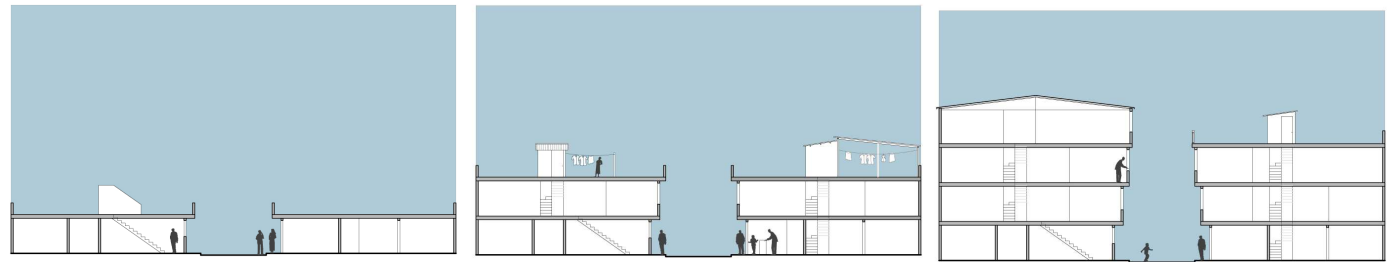

Modificación del perfil urbano con el paso del tiempo en Patio Bonito

Fuente: Juliana Arboleda Kogson, 2016

\section{¿CÓMO ENTENDER LA CALLE DE PATIO BONITO?}

Debido a que el tipo de calle que se construye en los barrios populares es diferente a la convencional, es necesario encontrar un tipo de bibliografía inspiradora, desde el punto de vista, que ayude a comprender la complejidad de los espacios que allí se erigen. Para este objetivo, el documento realizado por el geógrafo portugués Álvaro Domingues "A Rua da Estrada" que se refiere a la "calle de carretera" en Portugal (Domingues, 2009) resulta bastante pertinente puesto que la intención del texto, más que relatar la construcción literal de una calle, retrata de manera genuina las formas y procesos de su construcción en Portugal, en unas zonas donde la academia no ha advertido ningún proyecto ni ningún otro tipo de acercamiento. El retrato que hace de la Rua da Estrada no identifica su trazado, sino que relata todos los elementos que la componen: los diferentes tipos de viviendas que se alzan a sus costados, los cementerios que se ven desde la carretera e incluso los edificios comerciales y los antiguos monumentos. Todas las descripciones se efectúan en conjunción con los elementos que están presentes en el espacio público, desde artefactos temporales de ventas de frutas y verduras, hasta las paradas de autobuses que han quedado deshabitadas en la mitad del campo —o trozos de calle donde la acera se ha perdido-

La importancia de la colección, en términos urbanos, se confirma cuando Domingues explica que "contrariamente a la ciudad denominada 'consolidada', donde la estabilidad de las formas construidas y la regulación del edificio produce ciertas inercias y alguna resistencia a cambios bruscos, en la Rua da Estrada todo parece más plástico, más reversible y más disponible al cambio o a la novedad. Todo parece inestable y nunca terminado, será por la constante adición de elementos o por la transformación de los que allí ya se encontraban." Por lo tanto, podemos decir que es un tipo de calle que soporta bien el paso de los años y el crecimiento de las ciudades y poblados que se encuentran a su alrededor. El punto de vista de este texto sirve para comprender cómo en Patio Bonito y en muchos otros barrios populares, la calle es importante puesto que es la espina dorsal de los acontecimientos del barrio y de sus zonas aledañas. Por tanto, la calle debe ser entendida como un único edificio, pero no como un edificio especializado, sino más bien como un complejo de usos múltiples (tan de moda en las ciudades europeas) que permite una mixtura de usos y de personas. Es de alguna manera una invitación a conservar lo poco adoctrinado de la calle en lo popular dentro de una sociedad que tiende a la homogeneidad, las reglas y al orden. Es la informalidad de los acontecimientos la que permite que la ciudad se transforme constantemente y que, en vez de perder calidad, gane valor por la conjunción de las cosas y los elementos que la componen. Representa la calle de la contingencia.

Pero este punto de vista inspirador también puede abstraerse de textos más clásicos como el de "Towns \& Buildings" de Rassmussen (1969) o en el "Urbanisme et la règle des 7V' de Le Corbusier (1985) en los que la calle tradicional cobra un inigualable valor por ser la arteria principal de los acontecimientos de la ciudad. En el texto de Rassmussen la calle tradicional china es una mezcla de tiendas y talleres en los que los comerciantes exhiben sus mercancías, sus animales a la vez que producen actividades de abastecimiento, como la recogida de agua, o la ubicación de barberías y peluquerías y otras actividades de ocio. Son calles mixtas que se contraponen a las áreas residenciales donde no existen casi tiendas. La descripción ofrecida en este texto puede complementarse con lo descrito por Le Corbusier al hablar de la V4 en la que "sobre su recorrido se encuentran los servicios de la vida cotidiana: los alimentos (mercado, el charcutero, el carnicero, el panadero, etc.), las reparaciones (electricista, cerrajero, el farmacéutico, el dentista, el zapatero, la tintorería), las distracciones (el cine, las bibliotecas, las salas de conferencias, los cafés), la seguridad (la policía). Y así todo...," Son calles, como él denomina, donde se desarrolla la vida familiar, la calle de la tradición. Este tipo de calle mixta, llena de actividades diferentes es la armazón de los diferentes acontecimientos presentes en los barrios populares y que puede encontrarse de manera diversa en Patio Bonito. 
De esta manera la calle se entiende como un producto colectivo en el que tanto lo edificado como las personas tienen el mismo valor e importancia. Este valor es el que en definitiva ayuda a resolver los problemas que existen en el espacio público, como, por ejemplo, en los vacíos urbanos que registran menos uso, un tipo de espacios que no necesitan grandes cambios sino acciones de fina acupuntura que revitalicen y enriquezcan su configuración actual. Esta idea tiene que ver con lo que apunta una parte del texto de "Rehabitar la calle" (Monteys, 2012) al afirmar que "Domesticar la calle significa alejarla de la configuración de infraestructura y acercarla, mediante elementos y actividades, a su condición de lugar, asumiendo toda la complejidad que ello supone y que no puede reducirse a una simple fórmula de peatonalización. Si domesticar proviene de domus - casa en latín- podríamos decir que una 'calle domesticada' es aquella en la que uno se siente y actúa como 'en casa." La calle que aparece en Patio Bonito es pues una colección de alturas y secciones, de cajones que se ensanchan con el paso de los años y de espacios a la espera de pequeñas intervenciones. Una calle que se diferencia entre muchas otras existente en Bogotá por el tipo de elementos que la identifican y que se modifican rápidamente.

\section{ELEMENTOS DE LA IDENTIDAD}

Los elementos de la identidad pueden definirse como aquellos elementos presentes en el espacio público que son frecuentes en la arquitectura de los barrios populares y que desde la perspectiva tradicional deben regularizarse. Algunos parten de la idea de suplir una necesidad cotidiana y otros simplemente son el reflejo del carácter y la personalidad de los habitantes. La mayoría son elementos que forman parte del diario vivir de los barrios y las personas y que están presentes, tanto en barrios populares como en proyectos realizados por el Estado en las ciudades colombianas, pero también en ciudades como Roma, Barcelona o Marrakech según lo plantea Manuel Bailo en su tesis doctoral "Contra la indiferència: Catalitzadors de la Urbanitat" (Bailo, 2012). En todas estas ciudades existen una serie de elementos que califican y transforman el espacio y el entorno donde se han situado, muchos de los cuales, a pesar de su fragilidad o su poca escala - a pesar de ser transitorios o permanentes o de si son mínimos o secundarios- tienen una razón de ser dentro del espacio público y de ahí la necesidad de hacerlos visibles. Gran parte de los casos señalados por Bailo son espacios cotidianos en los que el interés está puesto no tanto en la arquitectura y la ciudad, como en los artefactos que se ubican y modifican lo público. Podemos decir que son ellos los que hacen que en la ciudad los proyectos formales e informales tengan un aspecto similar.

Estos elementos permiten realizar una radiografía de los espacios de uso y de la vida de cada una de las calles y en general de todo el barrio. Son interesantes puesto que, a pesar de que no son genuinos de Patio Bonito, pueden encontrarse en otras partes de la ciudad, expresan lo interesante y variado de lo popular y la manera en cómo se han mezclado particularmente allí. En muchos casos, son elementos que desde la academia se evitarían o bien se harían de forma consciente en la búsqueda por romper la monotonía de los proyectos, como cuando se trata de imitar intencionalmente el interior con los colores en la fachada, las alturas de las edificaciones o los elementos que forman una peculiar gramática arquitectónica. Estas acciones se complementan con el uso y la extensión de la planta baja de las viviendas sobre el espacio público y con la ubicación e incidencia de los vehículos de comercio y transporte temporal que en ella aparecen.

\section{El color y ornamento de la calle. El modelo de la calle fiesta}

El aspecto de las fachadas es una de las características que siempre llama la atención en un barrio popular. En el mundo ordenado y sistemático en el que se levantan de manera reglada la mayoría de las edificaciones actuales, encontrar escenarios donde lo individual prevalece y se demuestra en el plano vertical es bastante impactante. Más aún si se piensa, como lo han dicho Alberto Saldarriaga y Lorenzo Fonseca (Saldarriaga, 1984) que como cada fachada en un barrio popular es diferente de la anterior, la calle se convierte en la suma de fachadas desiguales. Esta suma contiene algunas fachadas que exhiben "a la vista" el bloque de ladrillo u hormigón y muchas otras que se han revestido y pintado e incluso se han decorado con formas geométricas. Lo que es visible en todas ellas son los sucesivos voladizos sobre la calle que se generan al levantar una planta, al ganar una nueva "parcela al aire", tal y como lo plantea Hernando Carvajalino (2004:103). También son parte del paisaje los cables eléctricos que van de un poste a otro alimentando las luminarias del espacio público y van conectando a la red eléctrica cada una de las viviendas. Puesto que fachadas hay tantas como dueños de viviendas, cada una se viste de manera diferente. Hay fachadas desnudas que muestran el ladrillo y tienen las ventanas y rejas también sin pintar. Hay otras que están desnudas pero que cuentan con todo el color en las carpinterías. Hay fachadas pintadas de un solo color con carpinterías a juego o que están revestidas de alicatado cerámico. Fachadas pintadas de colores estridentes. 


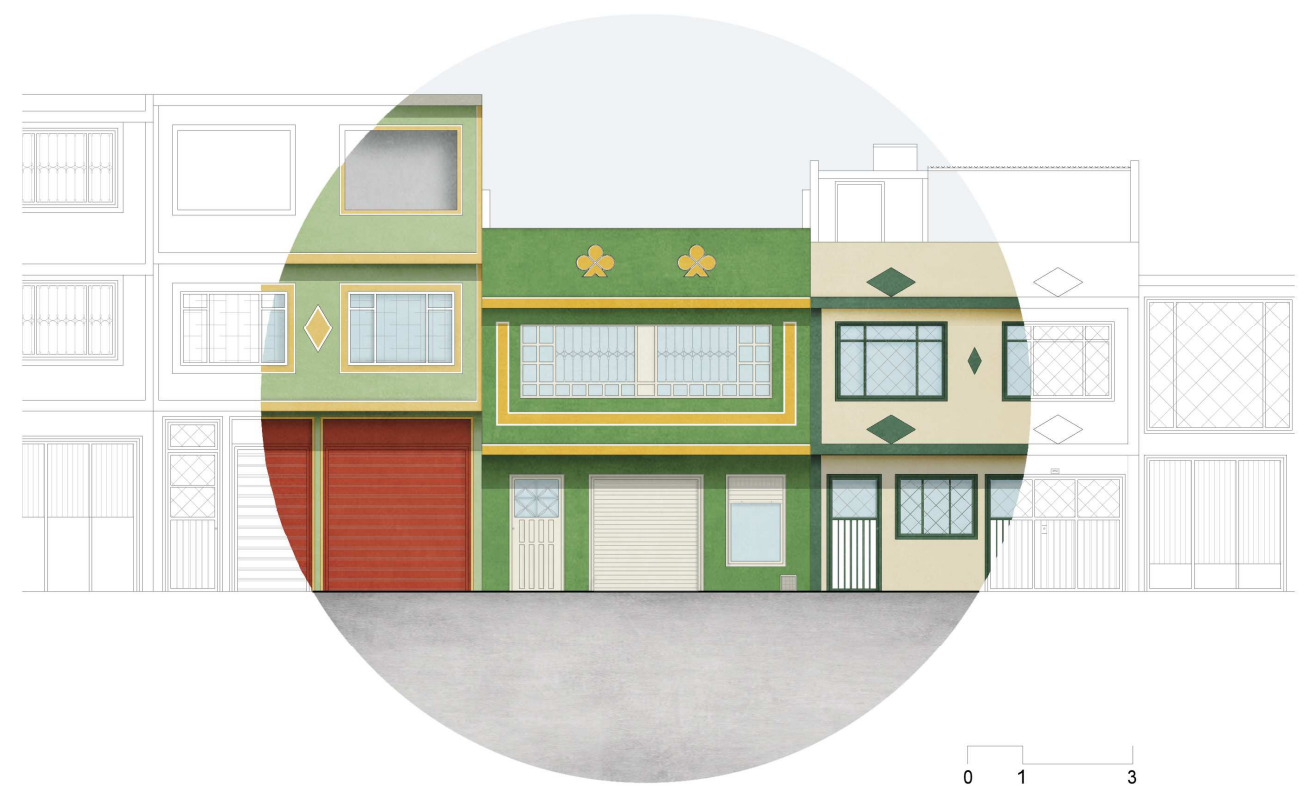

Fachadas decoradas en Patio Bonito

Fuente: Juliana Arboleda Kogson, 2016

Muchas otras que tienen un color de base y otro color con el que resaltar los cantos de los forjados, enmarcar las ventanas o hacer visibles los pilares estructurales. Los motivos ornamentales suelen ser variados, pero en el caso de Patio Bonito, llama la atención que tantas viviendas se decoren con rombos y que sean tan diferentes entre sí. Unas veces están situados de manera horizontal y otras de forma vertical, como elementos aislados marcando el centro del plano de fachada de la planta primera, o en parejas o tríos debajo de las ventanas o en el plano de remate de la construcción. En general pueden verse figuras puntuales como rombos, tréboles, estrellas y círculos - o grecas ornamentales más propias de las puertas de madera de una alacena- que se reproducen a gran escala en el exterior. Sorprende un poco saber que los rombos no son un elemento ornamental propio de Patio Bonito, sino que es una figura geométrica recurrente en los barrios populares en Colombia y en otros países Latinoamericanos como lo ha recogido y fotografiado Carvajalino. Se pueden observar ventanas metálicas pintadas y sin pintar, ventanas de aluminio anodizado pero nunca ventanas de madera. Todas ellas se complementan con rejas metálicas que, además de servir como elemento de seguridad, terminan de completar la decoración de las fachadas. Hay rejas de barrotes verticales, de barrotes horizontales, de mallas y formas geométricas. Hay otras que reproducen hojas, flores y otras formas naturales. Hay pocas viviendas que no tengan ninguna. La reja, como elemento de decoración se escoge en función del aspecto que se le quiera dar al conjunto, pero nunca se deja al azar.

Las calles decoradas son el telón de fondo de la vida que transcurre sin parar. Un telón que se compone del perfil discontinuo de las alturas de las viviendas que crecen y decrecen y que se enmarcan en el color del cielo bogotano - unas veces azul, otras veces nublado- . Son las fachadas en buena medida una muestra de la personalidad de los habitantes o del dueño de la casa. Es también la manera más genuina de conocer el sistema constructivo o de entender las etapas de crecimiento de la vivienda. Cara y ojos del exterior colectivo en un continuo cambio. Es importante tener presente que la decoración nunca dependerá del tipo de calle en que se ubique la vivienda, siempre será más o menos decorada dependiendo de sus propietarios. Tiene más que ver con la intención que ponen los habitantes al engalanar la calle para una fiesta, que en cumplir con una norma. Podríamos decir que se decora la fachada de manera "barroca", saturada de elementos, en consonancia con el interior de las viviendas en las que los colores estridentes también forman parte del repertorio. Una plasticidad homogénea entre el interior y el exterior. 


\section{El comercio y la plantas bajas}

El uso que damos a la planta baja y la incidencia de esta en el espacio público es, sin temor a equivocarnos, un tema pendiente de analizar en las escuelas de arquitectura y que tiene que ver fundamentalmente con la forma de observar la ciudad. La manera más usual de realizar dicho análisis concentra gran parte de los esfuerzos en revisar textos y archivos que registran la historia y las diferentes transformaciones de los lugares, en realizar fotografías y en hacer levantamientos de lo existente (alturas, elementos que lo conforman) pero pocas veces nos detenemos a observar el uso y la extensión de la planta baja. Siguiendo la metodología propuesta para el análisis de la calle Sant Pere Mitjá en Barcelona realizado por el grupo de investigación HABITAR-UPC (Monteys, 2013), se realiza un dibujo de una de las calles de Patio Bonito para entender la incidencia de lo público en la planta baja. Pero antes de pasar al dibujo es necesario advertir que en el barrio la calle, como elemento estructurador en la agrupación de las viviendas, se diferencia por el tipo de comercio que se ubica en la planta baja y por lo tanto por la extensión de lo público hacia el interior. La extensión de la planta baja difiere según el tipo de calle (si es de prevalencia peatonal, de comercio de barrio o una arteria de conexión con el resto de la ciudad), y por las actividades que se desarrollan en el espacio productivo de la vivienda. Por este motivo podemos encontrar calles con edificios completamente comerciales, calles con actividad comercial y servicios para el barrio, y calles peatonales en donde algunas plantas bajas usan o ceden un espacio para desarrollar una actividad productiva.

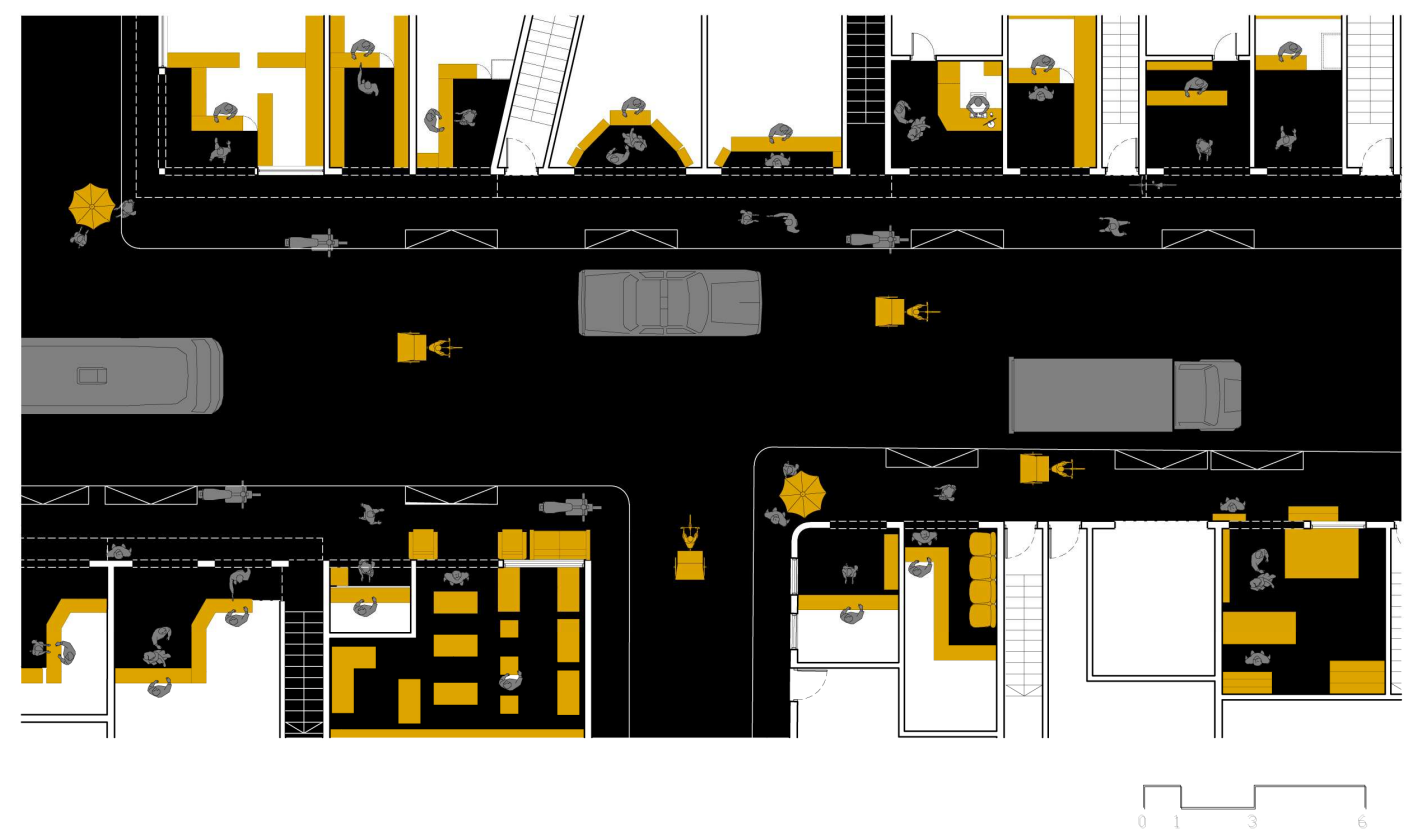

Extensión de la planta baja en la calle $38 \mathrm{C}$ sur de Patio Bonito Fuente: Juliana Arboleda Kogson, 2016

Las calles que desarrollan su actividad comercial pero que mantienen la residencia a partir de la planta primera, adquieren formas diferentes de atraer lo público hacia el interior. En ellas no hay una constante en la aproximación del peatón a los espacios interiores, sino que dependen del tipo de actividad para instaurar la forma de acceder y permanecer. Será diferente la manera y la cantidad de espacio disponible si entramos a una tienda de muebles o a una cafetería, en donde lo público invade hasta casi dos metros del interior, que cuando la actividad que se desarrolla en los "bajos" se despliega en forma de tienda de ropa, de papelería o de farmacia, puesto que una parte del género está "a la vista" pero otra parte está detrás del mostrador o en una bodega. Sin embargo, todas ellas son diferentes a las tiendas de abastecimiento en donde el límite entre el interior y exterior está dado por una reja metálica que, a modo de protección, filtra lo que sucede e impide que los clientes tengan contacto con el producto que se vende. En este tipo de calles el dibujo más eficaz para entender la manera en cómo se diluye lo público en el interior de los edificios, es el ampliamente conocido realizado por Giambattista Nolli en Roma, en el que lo público tiñe los espacios privados cuando inunda la planta baja, llevándose a su paso espacios interiores, aceras, personas y coches. Un tipo de calle que se complementa y se termina de activar con la aparición de artefactos y objetos del comercio ambulante que se ubican sobre las aceras, modificando el uso tradicional de estas y estirando la 
actividad comercial en todo su recorrido. Todas estas acciones y modificaciones son posibles ya que la planta baja permanece abierta y prolonga constantemente el interior hacia el exterior, y viceversa.

En buena parte de las calles de Patio Bonito la actividad comercial inunda no sólo la planta baja de la vivienda, sino todos los espacios disponibles en su frente más inmediato. Es así como sobre la acera se ubican aparadores comerciales, sofás, colchones, comedores, camas, y otros objetos para amueblar la casa o para construirla. Es decir, se usa la calle como aparador. También puede suceder que, si los objetos a vender son pequeños, estos terminen colgados de las fachadas de las viviendas llegando incluso a ubicarse muy cerca de las ventanas de la planta primera, como si se tratase de las abejas en una colmena. Una idea que difiere con la imagen limpia del espacio público que se observa en otras zonas de la ciudad, donde lo comercial se reserva al interior de las edificaciones y donde sólo los carteles promocionales explican lo que hay en cada tienda.

\section{Artefactos y espacio público}

Una atención especial merece los artefactos comerciales, que cumplen una función similar a los comercios de pequeñas dimensiones que tanto registraron e inventariaron los miembros del Atelier Bow-Wow (Mai, 2005) en Tokio, y que se instalan en el espacio público a modo de satélites contaminando tanto las aceras como las calzadas de la ciudad. Buena parte de ellos nacen de la mano de la economía sumergida, es decir, se ubican en la calle para generar ingresos, pero están fuera de los órdenes del trabajo y de la economía legal. A pesar de esta condición, suelen convivir en el espacio público con otros elementos propios del mobiliario urbano, de las actividades comerciales y del transporte normalizado que oferta la ciudad y son un complemento al uso que la gente hace de lo público. Estos elementos se dividen entre aquellos que están en constante circulación y otros que se quedan fijos en un tramo determinado de la calle. Suelen ser construidos mayoritariamente por sus dueños, con materiales ligeros para el cuerpo y con sombrillas y toldos para protegerse del sol. Comparten como particularidad la posibilidad de moverse y ubicarse en diferentes tramos de la calle desdoblando constantemente los espacios de uso de las aceras, y encuentran sus principales diferencias en la manera como los transeúntes se aproximan a ellos. Podemos encontrar algunos artefactos en los que la gente se ubica a su alrededor, como es el caso de los de comercio ambulante o las carretas de madera que, al tener a la vista la mercancía sobre un cajón, posibilitan un uso concéntrico de la porción de espacio público donde se ubican. Otros, en cambio, como los coches con espacio de venta en la parte trasera y los carritos de dulces, sólo permiten un acercamiento de los transeúntes por una de sus caras ya que cuentan con una parte frontal y otra posterior. Todos ellos en Patio Bonito tienden a ubicarse sobre las calles comerciales como la calle 38 sur, la calle 39 sur, la Alameda o en la Av. Ciudad de Cali, pero ya que pueden ser desplazados tienen la posibilidad de escoger el lugar del barrio donde son más pertinentes o donde pueden generar nuevas relaciones. Es tan fácil como empujarlos.

Los artefactos que son más visibles son aquellos que tienen un fin comercial. Se advierten inicialmente aquellos que portan una sombrilla y una superficie de venta en la que se ubica todo el género disponible y que normalmente están compuestos por una bicicleta con remolque, por un cajón de madera colocado sobre cuatro ruedas o por una mesita plegable como la que se usaría en la terraza de una vivienda. A pesar de estar diseñados para empujarse fácilmente, tienden a ocupar un lugar determinado dentro de la acera o de la calzada, siendo los únicos artefactos que pueden ubicarse en cualquier parte de la calle, configurando un tipo de mercadillo ambulante variado y colorido que anima el lugar en el que se asienta. Son un tipo de dispositivo común en todas las ciudades colombianas y siempre se ubican en las zonas de mayor actividad comercial, bien para la venta de objetos o de ropa o bien para ofrecer comida a quien transita. Al igual que en los carritos de dulces, la energía eléctrica se consigue ilegalmente por medio de un cable que se conecta a uno de los postes que están en el espacio público. Una energía que se usa para iluminar, pero también para conectar radios y altavoces con los que promocionar los productos disponibles. 


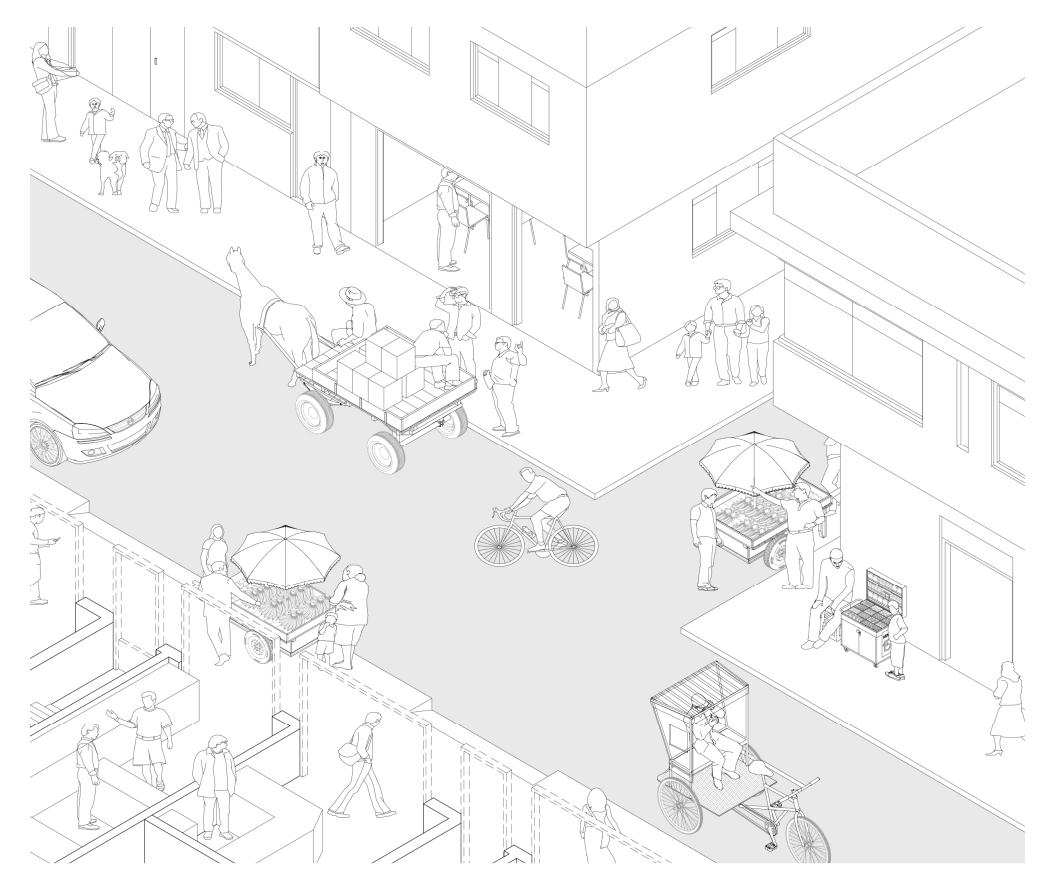

Axonometría de la calle y sus artefactos en Patio Bonito

Fuente: Juliana Arboleda Kogson, 2016

Un tipo de artefacto visible solamente en las aceras es el carrito para venta de caramelos comúnmente llamados como "carritos de dulces". Estos se componen de un cubículo de madera en el que su vendedor es una pieza más de su composición -al sentarse dentro o fuera de él- y que ofrece todo tipo de dulces y caramelos por unidades, de ahí su éxito. El cubículo suele tener un metro de ancho, por ochenta centímetros de fondo y casi dos metros de altura; y se dividen en dos partes, una primera de exposición situada de la mitad hacia arriba donde se organizan las golosinas en pequeños recuadros en horizontal, con patatas fritas, galletas y otros chuches empaquetados en las paredes verticales forrándolo todo, y una parte inferior, es decir de la mitad hacia abajo, que se reserva como almacenamiento. A pesar de que disponen de ruedas para su desplazamiento, tienen una posición fija dentro del espacio público intentando tener cercanía con las paradas de transporte, colegios, iglesias y otros equipamientos públicos que concentran mucha gente. Son de un único propietario y aunque no tienen ningún tipo de permiso ni posición sobre el suelo que ocupan, se respeta su ubicación. El carrito de dulces es el único dispositivo existente en el espacio público que en la actualidad se ha regularizado y se ha incorporado en el catálogo de elementos del mobiliario urbano de las ciudades colombianas, como por ejemplo, los instalados en la Av. Santander de la ciudad de Manizales en el año 2002.

De los muchos que pueden observarse en los barrios populares, el vehículo con espacio de venta es el más conocido, y a su vez el menos generalizado en el barrio puesto que, como no puede ubicarse en la acera sino que ocupa un trozo de la calzada, sólo se sitúa en lugares determinados. Estos vehículos traen mercancía de otros barrios hasta este punto de la ciudad y tienen como condición ser errantes. Son el típico artefacto comercial que puede verse en otras ciudades colombianas y en ciudades de Europa y Asia. Es uno de los medios de subsistencia más antiguos en la economía informal al permitir tener la mercancía guardada y posibilitar desplazarse rápidamente.

Existe un tipo de artefacto a medio camino entre los comerciales y los medios de transporte que se denomina carreta de madera, en los que la fuerza para el desplazamiento es de tracción animal, generalmente un caballo, al que se le engancha un remolque o cajón de madera, de un metro y medio de ancho por dos metros de fondo y paredes de treinta centímetros de altura, que descansa sobre cuatro ruedas. Son visibles en Patio Bonito y en otros barrios populares de la ciudad transportando desde materiales de construcción hasta frutas y verduras. Las carretas son más frecuentes en este barrio por su cercanía con la central de abastos "Corabastos", en la que consiguen parte de su género y, además, porque muchos de los transportistas viven en la zona más próxima al río Bogotá, cerca de Patio Bonito. A pesar de ser uno de los vehículos más antiguos en las ciudades colombianas, su uso es cada vez menos permitido puesto que las calles no están acondicionadas para su velocidad y maniobrabilidad. 

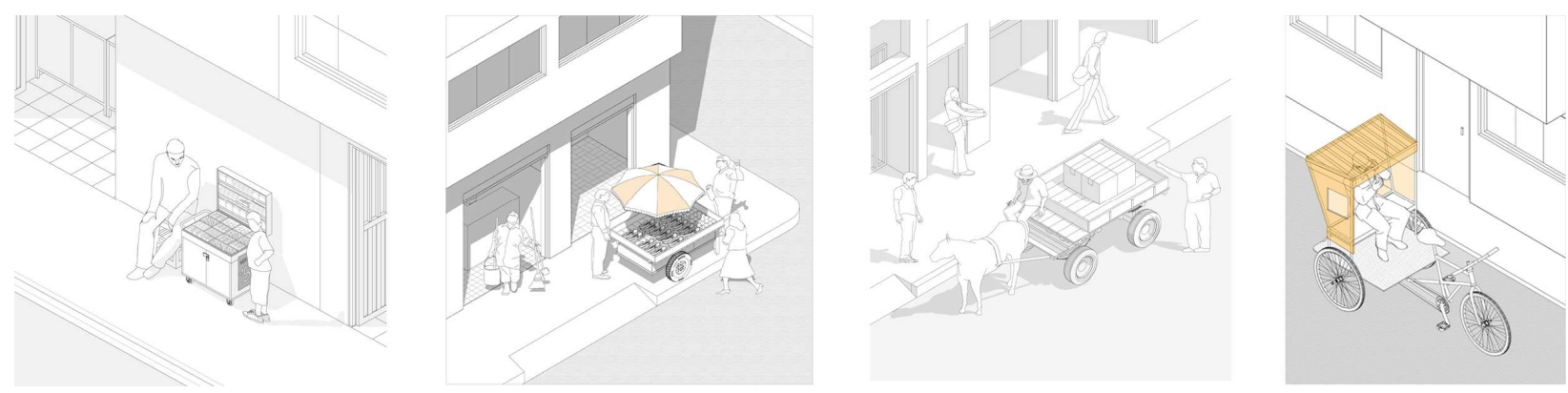

Artefactos presentes en el espacio público. De izq. a der. Carrito de dulces, cajón de madera sobre cuatro ruedas, carreta y bicitaxi

Fuente: Juliana Arboleda Kogson, 2016

Existe un último tipo de artefacto visible en el espacio público que es un nuevo medio transporte. Son los denominados bicitaxis, es decir, vehículos destinados al transporte de pasajeros y construidos bajo el principio de la bicicleta, accionados con tracción humana, con una capacidad de dos pasajeros sentados en la parte de atrás y un conductor. Este sistema de transporte, ahora tan común en los cascos antiguos de las ciudades europeas, fue innovador en Bogotá ya que apareció como complemento indirecto al sistema de transporte masivo, Transmilenio. Ya que los autobuses de Transmilenio sólo circulan por unas vías troncales definidas, el sistema debe apoyarse en unas rutas alimentadoras que recogen a los ciudadanos y los llevan hasta el portal de transporte más cercano. Pero, como las rutas alimentadoras no circulan por todas las calles y su frecuencia es insuficiente, muchos ciudadanos han optado por medios alternativos que los lleven hasta el sistema principal. Ante esta necesidad, nacen los bicitaxis como un sistema de transporte para el día a día (nunca para funciones turísticas), aunque uno sienta que está paseando desde el mismo momento en que lo aborda. Su uso se ha extendido por toda la ciudad y aunque aún no es reconocido por la administración local como parte del sistema de transporte público, forma parte de él ofreciendo una buena cantidad de unidades disponibles con un costo por trayecto muy reducido.

Todos los artefactos que hemos mencionado se instalan a primera hora de la mañana sobre las calles y a última hora de la tarde se recogen. Algunos se llevan hasta las casas de los vendedores, pero muchos otros (de pequeñas dimensiones) se guardan en las plantas bajas de los locales próximos a su ubicación. Estas ocupaciones temporales aluden directamente al concepto de post-it city con el que Martí Peran y su equipo (Peran, 2008) definían hace un tiempo una serie de acciones que se realizan en el espacio público de carácter comercial, lúdico o sexual que apenas dejan rastro pues aparece y desaparece constantemente. En la presentación del libro que recoge esta investigación ya se advierte que "los fenómenos post-it city ponen de relieve la realidad del territorio urbano como el lugar donde, de forma legítima, se solapan distintos usos y situaciones, en oposición a las crecientes presiones para homogeneizar el espacio público. (...) Desde otra perspectiva, las actividades temporales que infectan el espacio público con numerosos artefactos paraarquitectónicos permiten que la reflexión sobre la experiencia urbana reduzca su atención hacia lo minúsculo, corrigiendo así la arrogancia de la arquitectura tradicional". La incidencia de estos objetos en el espacio público, tal y como la plantea Peran, puede entenderse a partir de la obra del artista barcelonés Jordi Colomer (2005) quien en su proyecto "papamóvil" instala la maqueta del vehículo, en la calzada de un barrio en transformación en Barcelona esperando la respuesta de los peatones al verlo en la acera. Esta no es la primera vez que Colomer trabaja con maquetas de vehículos de transporte, pero si una de las que busca una relación directa entre el peatón y el objeto. Al instalar la maqueta en un espacio descontextualizado, fotografía la respuesta de quienes pasan por su lado. Algunos peatones caminan sin detenerse, otros se pasean intrigados a su alrededor y otros finalmente posan para la foto. Es una muestra de la alteración de lo público a partir de un elemento temporal concreto.

Todo lo anterior debería llevarnos pensar en la posibilidad de volver a instalar, de manera consciente, artefactos temporales que nos ayuden a configurar y alterar la calle. Decimos volver puesto que muchos de ellos han estado presentes históricamente en las ciudades, pero que con el paso del tiempo se ha desvirtuado su función perdiendo la capacidad de transformación cotidiana del espacio público. Los artefactos aparte de tener una función concreta permiten a los peatones ocupar la calle más allá de uso como infraestructura de recorrido para pasar a ser un espacio de encuentro. Esta idea puede asociarse a lo que sucede en los mercados dinámicos de Taipéi o con los coches de venta en las calles y rutas comerciales del sur de Italia recogidos por Peran o con los carritos para la venta de comidas rápidas fotografiados y analizados por Peter Cook (Cook, 2016) para implementar su uso en los parques en Londres o con las icónicas cabinas telefónicas inglesas. Más aún en Barcelona, por ejemplo, dentro de los elementos 
tradicionales del mobiliario urbano se han ubicado churrerías y quioscos de revistas, estas últimas tendientes a desaparecer según lo relatado en las noticias a inicios del año (Monteys, 2017) y que en la actualidad parecen asociarse más a los usos permitidos cuando la ciudad está de fiesta que en la vida cotidiana. Sin embargo, siguen apareciendo vehículos temporales de transporte, como los bicitaxis antes mencionados en Patio Bonito, pero aquí con un carácter turístico, o más recientemente los carritos para recoger chatarra. Todos estos elementos, propios de los barrios populares, forman parte en esta ciudad del espacio público reglado y consolidado conviviendo sin problemas con otros elementos tradicionales del mobiliario sin generar problemas y permitiendo construir un espacio público democrático que se modifique a diario.

\section{COMO EXTRAPOLAR LA EXPERIENCIA DE PATIO BONITO?}

Para encontrar la forma de aprender de la experiencia urbana de los barrios populares, como el caso relatado de Patio Bonito, es necesario volver a la discusión inicial acerca del espacio público que actualmente se construye en las nuevas zonas de crecimiento de Bogotá. Para ello nos valdremos de lo explicado por Richard Sennett (1997) al referirse que en la actualidad "al planificar un distrito, los urbanistas situarán la escuela y las viviendas en el centro en vez de en su periferia, donde la gente podría entrar en contacto con extraños. Cada vez más, se vende a los compradores una comunidad planificada con verjas, puertas y guardias como si ésa fuera la imagen de la buena vida." La explicación genérica que Sennett nos relata es tal vez la definición más precisa de lo que sucede en los nuevos conjuntos de vivienda que se construyen para albergar las viviendas de interés social, donde se ha prestado atención en construir múltiples bloques de vivienda de gran altura, que se complementan con equipamientos urbanos y con unas calles de grandes proporciones pero que no han advertido que la especialización de las partes no confiere calidad a lo público. Un caso reciente que nos sirve para ejemplificar esta idea es lo que sucede en el proyecto de vivienda "Ciudad Verde", un polígono de vivienda edificado en el municipio de Soacha, aledaño a Bogotá, en el que se busca albergar 49500 familias en una "una pequeña ciudad dentro de la ciudad" sobre un territorio que se extiende en 328 hectáreas.

Para hablar de la especialización del uso del suelo es necesario observar la planta general del macroproyecto. En ella se distinguen fácilmente las áreas verdes, los parques infantiles, los colegios y guarderías, los centros comerciales y las zonas destinadas a la vivienda. Por una parte, es evidente que la urbanización de toda la zona previa a la llegada de los habitantes, posibilita el acceso a vías mejor construidas y la conexión con los servicios públicos, además de generar los equipamientos colectivos necesarios para la vida diaria (colegios, guarderías, iglesias, entre otros). Lo que se pierde con este modelo, sin embargo, es la vida de la calle al no existir comercio en las plantas bajas, una situación que evita los recorridos a pié y que confina las actividades de este tipo al centro comercial. Esto sumado a que la calle y el espacio público que se tiene una proporción desmedida a nivel peatonal, siendo hasta de cincuenta metros el espacio libre construido entre conjuntos de bloques de edificios, si se suman los antejardines, las aceras y las vías de circulación. La monumentalidad creada en el espacio público se contradice rápidamente al observarse las rejas metálicas que confinan cada conjunto urbano. Al final la calle creada es un lugar de nadie puesto que las actividades colectivas se desarrollan en unas zonas delimitadas dentro de cada conjunto, en medio de las circulaciones de coches y los aparcamientos al aire libre.

Si logramos entender que la importancia de los escenarios urbanos no está solamente en la vivienda, sino en el espacio público que se ha creado a partir de lo colectivo, podremos definir de mejor manera la calle en las nuevas urbanizaciones, así como intervenir en los existentes a partir de pequeñas microcirugías en las que la calle y sus elementos urbanos ganen orden y jerarquía o donde simplemente se aborden las esquinas para construir sobre lo construido. Volver la mirada a lugares como Patio Bonito, como lo están realizando las universidades, nos da una pista acerca de cómo la academia puede ayudar a resolver los vacíos que deja la urbanización popular. Las nuevas visiones obligan a actuar no solamente sobre la vivienda, sino que debe apreciarse la calle como un proyecto que está a la espera de que alguien encuentre la mejor manera de ubicar y aprovechar los artefactos y objetos que la componen para darle más valor al espacio público. Pero sobre todo este tipo de ejercicios debe servir como ejemplo para corregir el error de seguir extendiendo la ciudad sobre la sabana que la rodea. Ciudades donde el verde abunda, pero en cambio faltan rutas y paradas de autobuses, donde la agrupación de bloques de vivienda resuelve rápidamente unidades habitacionales pero que en cambio se crea un espacio público anodino y desangelado que nunca se usa. No se puede perder la ruta e imaginar que lo nuevo es lo que necesita mayor atención, siempre lo existente debe llevarse la mejor parte. 
La búsqueda por hacer un protocolo que permita extraer las mejores enseñanzas del espacio público y concretamente de la calle realizada en los barrios populares está aún por comenzar y es un campo lleno de oportunidades. Acciones como el ejercicio "Repensando la informalidad" (MIT DUSP+UNAL, 2014) realizado por la Universidad Nacional de Colombia, Sede Medellín de manera conjunta con el MIT son una apuesta en este sentido al intentar encontrar nuevas prácticas contemporáneas para la intervención espacial en las zonas más consolidadas de nuestras ciudades. Al respecto es oportuno el comentario de Sennett en su libro "l'espai public" (2014) donde opina que "La batalla ideológica nos parece poco importante a la hora de pensar cuál debería ser el futuro de las ciudades. Deberíamos responder a la pregunta de cuáles deberían ser las normas de su diseño, más que no si debería haber normas de algún tipo. Queremos normas que abran el hábitat al cambio, y no que lo estabilicen, y eso quiere decir establecer, en la práctica profesional, un conjunto de regulaciones en la planificación o del diseño de los edificios que son muy prescriptivos, y por tanto evitan la adaptación y evolución a través del uso. Queremos estructuras que tengan la misma obstinación de auto-revisarse que tiene la cibernética de Norbert Wiener. En un sistema cerrado, esta cantidad de cambios internos son imposibles. Por el contrario, en un sistema abierto, la forma construida demuestra que es capas de metamorfosearse. Para decirlo de manera abstracta, la forma construida debería soportar la fuerza transformadora del tiempo. El concepto de construir a partir de un contexto puede hacer que esta abstracción cobre vida." La invitación es por tanto a pensar que, en la ciudad la calle antes de haber prescrito, está a la espera que alguien la intervenga con pequeñas acciones de acupuntura que la mejore y aporte valor a sus cualidades. Volver la mirada a lo popular significa comprender que "los menos privilegiados tienen una lección que enseñar, hay vida, comunal e individual, más allá del centro comercial' (Zabalbeascoa, 2010). El patio también puede ser bonito.

\section{BIBLIOGRAFÍA}

ARBOLEDA KOGSON, J. (2016). Patio Bonito. Un barrio sin proyecto. Tesis Doctoral. Barcelona: Universidad Politécnica de Cataluña. Departamento de Proyectos Arquitectónicos.

BAILO ESTEVE, M. (2012). Contra la indiferència: Catalitzadors de la Urbanitat. Tesis Doctoral. Barcelona: Universidad Politécnica de Cataluña. Departamento de Urbanismo y Ordenación del Territorio.

BORTHAGARAY, A. Director. (2009). ¡Ganar la calle! Compartir sin dividir. Buenos Aires: Ediciones Infinito.

CARRASCO, R. R. (2004). Barrios Marginales en el ordenamiento de Bogotá. Bitácora Urbano- Territorial, 8,56 .

CARVAJALINO, Hernando. (2004). "Estética de lo popular: los engalles de la casa" En: Barrio Taller. Serie Ciudad y Hábitat \#11

COOK, P. (2016). Motive 7. Can learn from silliness? En: Architecture workbook: design through movie. (187-209). Chichester, West Sussex: Wiley.

DOMINGUES, A. (2009). A Rua da Estrada. Oporto: Equaçoes de Arquitectura. Dafne Editora

ESCALLÓN GARTNER, C. Et al. (2008). El hábitat popular. ¿Cómo cambiar el paradigma?. En XXI Bienal Colombiana de Arquitectura. Construyendo valor con la poética del lugar (256-261). Bogotá: Sociedad Colombiana de Arquitectos SCA.

FUNDACIÓN SOCIAL; CORPOGES. (2010). Balance de procesos de planeación del territorio. Patio Bonito. 2002- 2010. Resultados, logros y aprendizajes. Bogotá

GARCíA DONATO, A. (2011). Por el derecho a construir la felicidad en Patio Bonito. Una experiencia de planeación y gestión colectiva que se construye día a día. Bogotá: CORPOGES. Corporación Grupo Enlace Social

IMAI, K. (2005). Pet architecture guide book. Nakano: World Photo Press.

LE CORBUSIER. Et al. (1985). Le Corbusier: Oeuvre Complète. Zurich : Éditions d'Architecture 
MIT DUSP+UNAL (2014). Repensando la informalidad. Estrategias de Co-producción del Espacio Urbano. Medellín- Comuna 8. Medellin: MIT DUSP+UNAL

MONTEYS, X. Et al. (2012) Rehabitar en nueve episodios. Madrid: Ministerio de Fomento

- (2013). Rehabitar: La casa, el carrer i la ciutat: Barcelona com a casa d'estudi. Barcelona: UPC, Departament de Projectes Arquitectònics.

— (2017). Xurros necessaris. Artículo de prensa. Quadern de Catalunya. El Pais. 08/01/2017.

PARIAS DURÁN, A. (2012). Configuración urbana y vivienda popular. En: Samper, Germán; Samper, Marcela Ángel y O’Byrne, María Cecilia. Casa+ Casa+ Casa = ¿ciudad?: Germán Samper, una investigación en vivienda $(74-85)$. Bogotá: Uniandes.

PERAN, M. (2008) Post-It City: Ciutats Ocasionals = Ciudades Ocasionales = Occasional Urbanities . Barcelona: Diputació de Barcelona

RASMUSSEN, S. (1969) Towns and Buildings: Described in Drawings and Words. Cambridge (Mass.): The M.I.T. Press

SALDARRIAGA, Alberto; FONSECA, Lorenzo. (1984). Los colores de la calle. Bogotá: Carlos Valencia Editores

SENNETT, R. (1997) Carne y piedra: El cuerpo y la ciudad en la civilización occidental. Madrid : Alianza

- (2014) L'espai public. Un sistema abierto, un proceso inacabado. Barcelona, Arcadia Editorial

ZALBABEASCOA, Anatxu. (2010). "Arquitectura y pobreza” En: Blog del tirador a la ciudad. El País

FUENTES ELECTRÓNICAS:

COLOMER, Jordi. "Propotipos 2" (2004) y Papamovil (2005). http://www.jordicolomer.com (Consulta: 25/04/2017) 